\title{
Wearable Technology and Electronic Visualisation of Artistic Process: Google Glass meets Fine Art Painting
}

\author{
Gretchen Andrus Andrew \\ 4 Glassworks Studios, Basing Place, \\ London E2 8AB, United Kingdom \\ gretchenandrus@gmail.com | @underscoreg.com | @gretchenandrew
}

\section{INTRODUCTION}

Fine art oil painter Gretchen Andrus Andrew has been using Google Glass to create film visualisations of her artistic process. A live demonstration will discuss Google Glass and wearable technology generally while showing specifically how Gretchen Andrus Andrew uses it in her artwork.

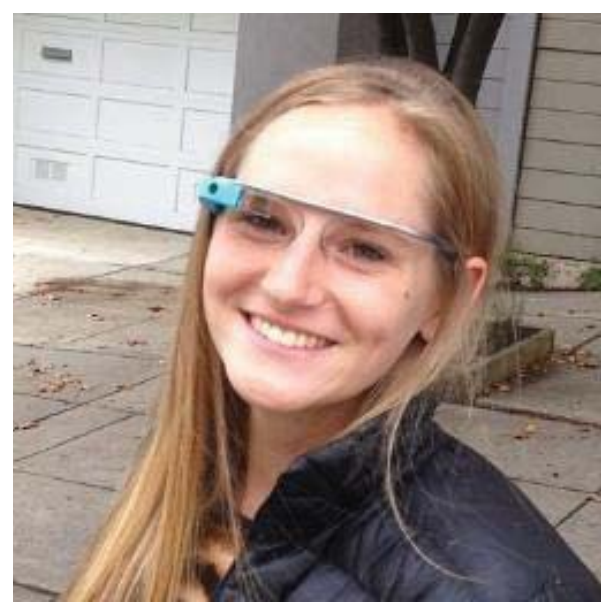

Figure 1: Google Glass as shown on artist Gretchen Andrus Andrew

\section{DESCRIPTION OF SYSTEM AND TECHNOLOGY}

Google Glass is Google's wearable technology product that sits on the face like glasses. Google Glass is not yet publically available. Google selected artist Gretchen Andrus Andrew to experiment with Google Glass's artistic potential.

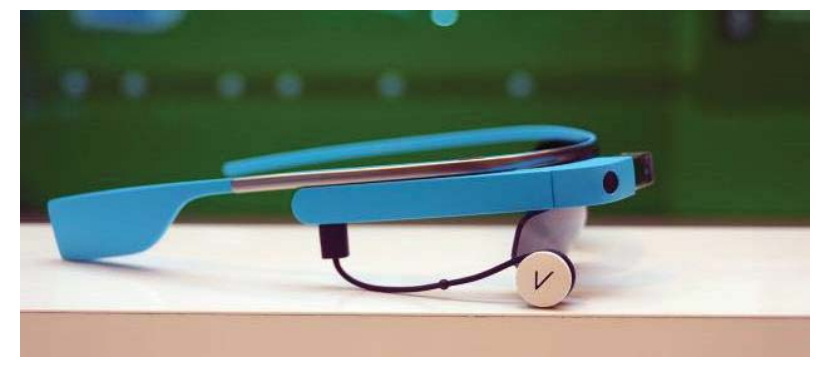

Figure 2: Google Glass device with ear bud

\section{FEATURES AND SCENARIOS THAT WILL BE DEMONSTRATED}

Day 1 of the conference is spent recording videos and photographs using Google Glass. These will be inspiration inputs for the following day's painting.

Day 2, live painting is set up. A new large-format oil painting is made on site. The painting process is recorded and shared with Google Glass.

Day 3, final presentation: demonstration of Google Glass's key features (search, call, text, direction, photo, video).

Examples of how Google Glass is used in artistic process including video produced with the Metropolitan Museum of Art New York and a video showing the creation of the painting made on days 1 and 2.

\section{SIGNIFICANCE TO AUDIENCE}

Wearable technology like Google Glass fundamentally changes the nature of the digital content we can collect and analyze. Hands free, point of view recording will have impacts beyond the arts. 


\subsection{Privacy concerns discussed}

Cultural and political practices question the impact of wearable technology products on privacy. Artists have always had an interesting relationship to their own and other's privacy. Artists frequently tap traditionally private content for creative production.

\subsection{Shareable visual process}

Sharing process communicates intention, an important aspect of art compared to ordinary objects. Google Glass is investigated as a tool that can be used to share creation processes in an attempt to deepen the artist's and the viewer's relationship to the created artwork. The processcreator and process-consumer relationships are examined as a model for business, design, and conflict resolution.

\subsection{Link between new digital media and traditional painting medium}

How does technology like Google Glass impact old media like painting? This demo will present a way the old and new can strengthen each other while questing the impact on the arts and culture.

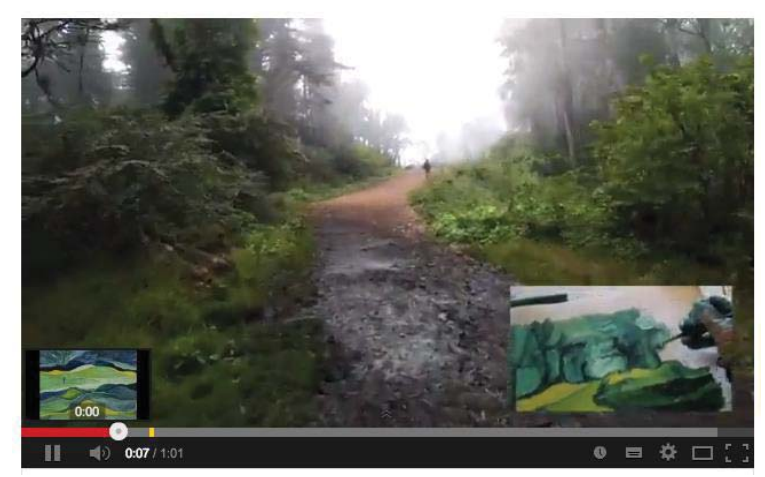

Figure 3: Still from video recorded using Google Glass (Video recorded painting being made alongside recorded inspiration)
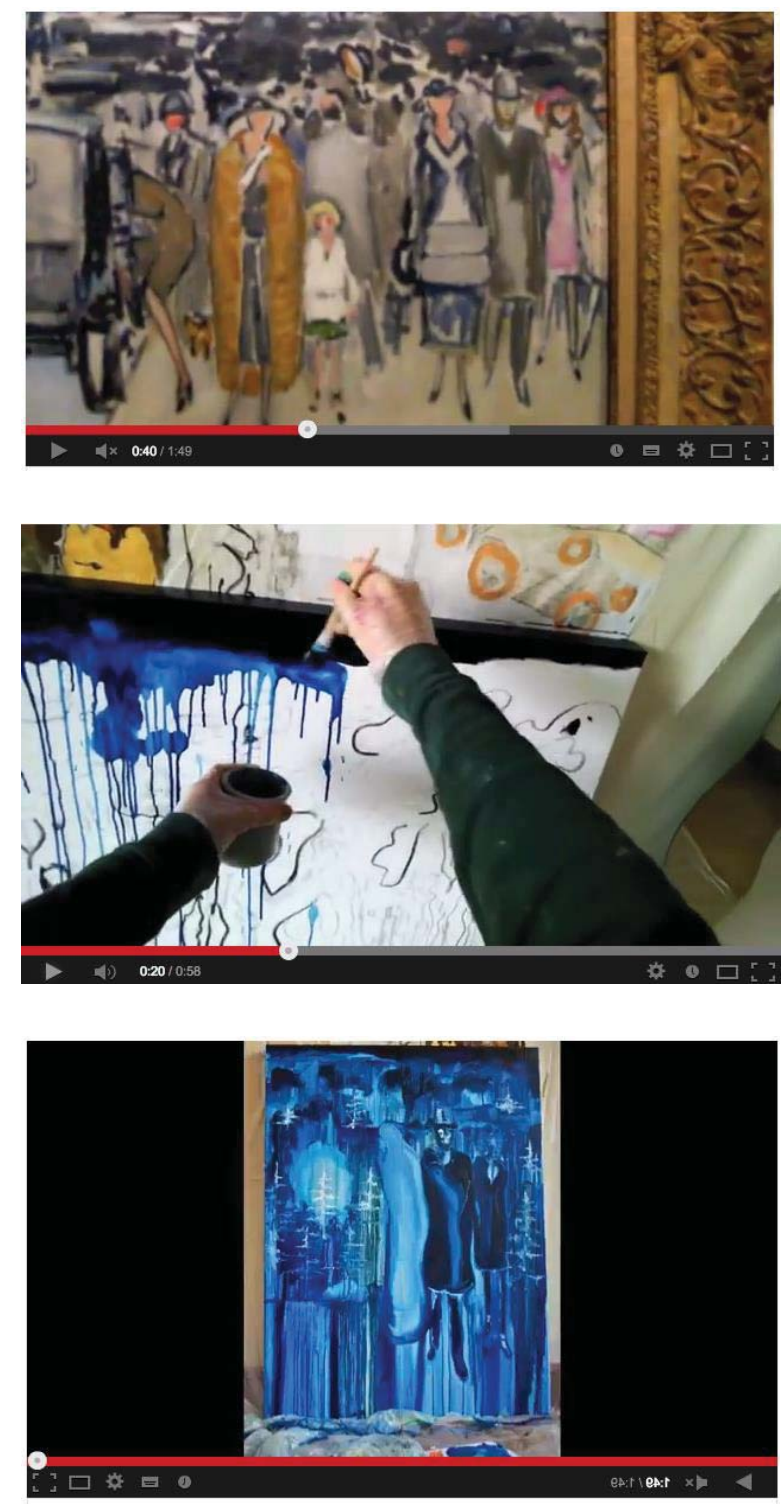

Figure 4 (1-3): Video recorded with Google Glass used in collaboration with the Metropolitan Museum of Art, New York (Video links a piece in the Met's collection with a new work being made)

\section{REFERENCES}

Stimler, N., and Andrew, G. A. (2014) Making New Art Inspired by the Met's Collections with Google Glass, The Metropolitan Museum of Art, New York, USA. http://www. metmuseum.org/about-themuseum/museum-departments/office-of-thedirector/digital-media-department/digitalunderground/2014/making-new-art (retrieved 18 May 2014). 